\title{
LAS INDISCIPLINAS SOCIALES. UNA EXCUSA PARA NO MIRAR ALLÁ
}

CELIA M. RIERA VÁZQUEZ ${ }^{1}$ / IDALSIS FABRÉ MACHADO²

\section{RESUMEN}

En los últimos años se ha venido empleando en Cuba desde las instituciones representativas del control social formal, el término indisciplinas sociales para dar cuenta de comportamientos socialmente reprochables. E debate sobre esta problemática queda en los estrechos marcos del instrumentalismo y las posturas correctoras del comportamiento desviante. Los vacíos relativos a la normatividad social dan al traste con enfoques reactivo represores, que no parten del análisis del ordenamiento de la sociedad, de sus formas diferenciadas de organización; sino de una visión que redunda en la creación de estereotipos y la estigmatización de determinadas conductas y grupos. De ahí la necesidad de que las Ciencias Sociales aporten un marco conceptual para el análisis crítico del tema.

Palabras claves: indisciplinas sociales, control social, socialización, desviación social.

\footnotetext{
${ }^{1}$ Doctora en Ciencias Sociológicas. Asociada al Centro de Estudios Comunitarios, Facultad de Ciencias Sociales, Universidad Central "Marta Abreu" de Las Villas. Carretera a Camajuani km 51/2. Código Postal 50100, Santa Clara, Provincia de Villa Clara (Cuba). Correo electrónico: celiam@uclv.edu.cu

2 Máster en Desarrollo Social. Asociada al Centro de Estudios Comunitarios, Facultad de Ciencias Sociales, Universidad Central "Marta Abreu" de Las Villas. Carretera a Camajuani km 51/2. Código Postal 50100, Santa Clara, Provincia de Villa Clara (Cuba). Correo electrónico: idalsisFM@uclv.edu.cu
} 


\section{SOCIAL INDISCIPLINE. NO EXCUSE FOR A LOOK BEYOND}

\section{ABSTRACT}

In recent years it has been used in Cuba from the representative institutions of formal social control, social indiscipline term to account for socially reprehensible behavior. The debate on this issue is in the narrow framework of instrumentalism and corrective postures deviant behavior. Empty concerning social norms give the kibosh on reactive approaches repressors, not based on an analysis of the ordering of society, its different forms of organization; but a vision that leads to stereotyping and stigmatization of certain behaviors and groups. Hence the need for social sciences provide a conceptual framework for critical analysis of the topic.

Keywords: social indiscipline, social control, socialization, social deviance.

\section{Introducción}

La sociedad es un mecanismo complejo y dinámico que se constituye como espacio de confluencia de las relaciones entre los individuos. Se apoya en varios instrumentos para lograr la homogenización del accionar de los sujetos, en busca de mantener un equilibrio y estabilidad a su interior, en resumen mantener la cohesión social. Desde la perspectiva de Castells (1997) la cuestión social da cuenta de un estado de lo social que remite a la complejidad de su cohesión y los modos de prevenir y solventar el riesgo de su fragmentación por ello, tal estado, reta a la potencialidad de una sociedad para mantenerse cohesionada en tanto Nación. A ello se incorpora la puesta en cuestionamiento de la validez y funcionalidad de las normas como garantes del orden, el equilibrio y la cohesión social.

La tendencia a la "normalidad" está latente en las más incipientes expresiones positivistas y estructural-funcionalistas, que apelan a un orden preestablecido en que el sujeto queda relegado a un rol secundario de adaptación y sometimiento. 
El equilibrio social descansa en la lógica funcional de procesos como la socialización y el control, garantes del amalgamiento de la sociedad desde una perspectiva donde toda aquella conducta que se aparte de lo "colectivamente legitimado", constituye expresión de desviación social.

Lo imprescindible entonces, es examinar desde una perspectiva crítica y en franco posicionamiento dialéctico, qué mecanismos median en la construcción social de aquello a lo que comúnmente identificamos como un comportamiento desviado. Lo cierto es que la desviación no se produce en abstracto, sino de acuerdo a aquellos estándares sociales que establecen y pautan lo "antisocial" de cada conducta de individuos y grupos que interactúan en la sociedad.

La sociedad no puede renunciar a su función reguladora, que se expresa en ciertos mecanismos más o menos coercitivos o persuasivos que compulsan a los sujetos a actuar de acuerdo a los principios, valores y normas por ella establecidos. Este proceso está mediado por prejuicios, estereotipos y sesgos que lamentablemente redundan en concentrar la atención en determinados grupos o sectores sociales a los que históricamente se les ha identificado como portadores de esa condición desviante. Nos referimos a los pobres, marginados, homosexuales, hippies en fin, cada época histórica ha hecho sus aportes al respecto.

En este sentido, dichas manifestaciones del comportamiento, desajustadas respecto a un entorno social determinado, se corresponden con las expresiones de aquello que se define como indisciplinas sociales. Este término se ha venido empleando en los últimos años en Cuba con un fuerte sentido instrumental y operativo y ha sido incorporado, tanto desde el discurso oficial como desde la práctica de aquellas instituciones que en su función social se reconocen como agentes del control social formal.

Esta perspectiva se corresponde con los postulados de la Sociología clásica, la cual concibe:

[...] el bien, no como la armonía con el orden del mundo o con las leyes divinas y ni siquiera con la producción de un orden que ponga freno a las pasiones y a la violencia, sino como la contribución de un actor- o mejor de un órgano- al funcionamiento del cuerpo social... (Touraine, 1993, p. 345). 
Desde la política preventiva en Cuba y su implementación, las denominadas indisciplinas sociales se han convertido en centro de atención en tanto fenómeno negativo emergido en nuestra sociedad contrario a su naturaleza y asociado al deterioro de determinados valores.

Existe una estrecha relación entre la formación de valores y la configuración de fenómenos como el de la indisciplina social, como expresión de una desviación en el comportamiento. Esto ha sido abordado desde la literatura que en los campos psicológico, sociológico y criminológico ha trabajado el tema.

En este particular se impone dar cuenta de determinados aspectos contextuales de carácter coyuntural por las que ha atravesado nuestro país, y que impusieron una nueva jerarquía en los valores tradicionalmente asumidos por el proyecto social cubano (Alfonso, 2008) dada una nueva cotidianeidad que requirió de una organización racional que marcara una diferenciación respecto al disciplinamiento.

Se alude al considerado Período Especial que vivió Cuba, enmarcado en la década del 90 del pasado siglo y las consecuencias asociadas al mismo en las diferentes esferas de la sociedad. Algunos autores cubanos, entre ellos Mayra Espina (2003) denominan a este período con el término reajuste, identificando así tanto al proceso de crisis de todos los órdenes que siguió al derrumbe del campo socialista como a las medidas que se han tomado para enfrentarla, o sea el reajuste es la crisis más su enfrentamiento. A criterio de Miguel Limia (2002) el período especial ha traído consigo una profunda modificación de las estrategias vitales de cada persona, pues ha significado entre otras cosas una crisis de los fundamentos de la vida cotidiana.

Sumado a ello se articulan las negativas consecuencias derivadas del proceso de globalización neoliberal que, en franca refuncionalización de los ideales capitalistas, ha apostado por la exacerbación del individualismo, el consumismo depredador y la mercantilización de la vida. A ello que no está ajena Cubana. Estas condiciones, tanto externas como internas, que se generaron a partir de la década del 90 propician estados críticos de las históricas estructuras que modelaban del orden social hasta entonces, de las 
instituciones clásicas de socialización, se produce la emergencia de situaciones de incertidumbre que son percibidas y vivenciadas por los sujetos individuales y colectivos con un consecuente impacto en los planos axiológico y comportamental.

Al respecto, Limia (1997) expresa que:

Los cambios ocurridos en la sociedad cubana a lo largo de la revolución han modificado profundamente la fisonomía, status y roles de las clases sociales y de los grupos socioprofesionales, así como su dinámica interna e interrelaciones; además, también se han plasmado de forma diferente en las distintas generaciones de cubanos actualmente existentes (p. 47).

Las preguntas claves pasan por el sentido de la vida vivida y por vivir, los grupos de referencia realmente significativos y solventes, así como por los medios hábiles (sociales o individuales) para llevar a efecto los planes de la vida reformulados en las presentes circunstancias sobre otros fundamentos que los tradicionales del proceso revolucionario (Alonso et. al, 2004). De ahí que a nivel de individualidad se esté configurando una marcada crisis de identidad psicosocial de carácter socio histórico.

La trascendencia de los argumentos anteriores no puede convertirse en excusa para enmascarar conductas que entrañen procesos desintegradores de la sociedad cubana pues, los valores nos identifican precisamente por su universalidad y carácter imperecedero. Ahora bien, sí es preciso contextualizar los análisis, atemperarlos a una realidad que ha cambiado en la que la concreción de estos valores, su materialización, se proyecta hoy en un sistema de relaciones sociales que los reconstruye y emergen en códigos diferentes.

La indisciplina social, expresión de conflicto entre la conducta socialmente aceptada y la manifestada, constituye un fenómeno explícito en la sociedad cubana actual. Ella es expresión de una dinámica social de ruptura de determinados órdenes a consecuencia de transformaciones socioeconómicas iniciada en los años 90 del pasado siglo, como ya se apuntaba. 
Es una realidad que se percibe diariamente en el espacio público como la violación de determinadas normas de convivencia social; sin embargo, es también un fenómeno que muchas veces se sobredimensiona y bajo cuyo rótulo se etiqueta a aquellos con quienes sencillamente no se comparte un modo de comportamiento, porque se parte de preceptos culturales residuales que pueden estructurar prácticas estigmatizadoras y excluyentes-.

Como bien expuso Mills (1996), interpretando a Parsons, “...las gentes admiten con frecuencia las mismas normas y esperan que todos se atengan a ellas. En la medida en que lo hacen así, su sociedad puede ser una sociedad ordenada" (p. 49).

Desde este posicionamiento, la problemática generacional, la coexistencia de diferentes sujetos económicos, las contradicciones entre capital cultural y económico, entre otros elementos, desempeñan un papel muy importante para el posicionamiento frente a las llamadas indisciplinas sociales ¿Qué pasa con los sujetos económicos emergentes, por qué valores están apostando? ¿Cómo posicionarse positivamente en medio de la coexistencia de varias generaciones con puntos de partida y referentes diferentes? ¿Cómo abordar las divergencias entre una misma generación cuyos patrones socioculturales la hacen tan heterogénea en medio de la dinámica de la sociedad cubana de estos días?

Todas estas interrogantes y muchas otras que pudieran surgir, se convierten en premisas para las Ciencias Sociales en Cuba hoy. Ante el reto de contribuir desde el análisis, el debate y la reflexión teórica al diseño de políticas que vayan más allá del acercamiento a las expresiones de fenómenos como las indisciplinas sociales e incidan en sus condicionantes.

\section{Acción y conflicto sociales para el análisis del término indisciplina social}

La acción social es la forma de comportamiento dentro de la sociedad que caracteriza a cada individuo, tiene un trasfondo subjetivo y está fuertemente condicionada por el proceso de socialización. En tanto permeada de valores, creencias, hábitos, vigentes en la sociedad y marcada además por 
su contexto; está estrechamente vinculada con lo que desde el pensamiento social se ha definido como conflicto.

El conflicto social es una categoría muy amplia, está integrada por casi todos los fenómenos que se dan al interior del entramado social. Este no puede ser entendido solamente por divergencias y luchas; lleva implícito el consenso, la integración; para que exista deben existir sujetos individuales o colectivos que estén dispuestos a enfrentarse de forma abierta o de manera más sutil .Por lo que es posible plantear que son diferentes los modos en que el conflicto se manifiesta en la sociedad, va desde la lucha de clases hasta diferencias al interior del grupo.

Las acciones sociales son intencionales, tanto a nivel grupal como individual, orientadas hacia fines concretos subjetivamente deseados y objetivamente condicionados, matizadas tanto por el conflicto como por el consenso. El individuo expresa con su comportamiento preferencias y metas que aspira alcanzar. Cuando este comportamiento es contrario a las normas y leyes socialmente establecidas se puede inferir que se habla de indisciplina social.

La indisciplina social aparece entonces en la sociedad, como expresión de conflicto social donde las acciones sociales de uno o varios individuos se manifiestan en contradicción con las esperadas por la sociedad en general. Surge como resultado una contradicción entre el comportamiento socialmente aceptado y el expresado, la cual debe ser resuelta por mecanismos de regulación social como el control, puede primar el enfoque reactivo represor o el preventivo proactivo.

La conflictividad de la condición desviante latente en la indisciplina social no puede ser entendida en términos simplistas como mero desajuste o ruptura con el orden establecido. Sin dudas son atributos asociados a aquellos comportamientos etiquetados como indisciplinas sociales pero su conflictividad no es más que expresión de que constituyen fenómenos comunes a cualquier tipo de sociedad. 
Contrario a las ideas que se sustentan en la doctrina del contrato social, en franco posicionamiento defensista y desde posturas conservadoras; el análisis de las indisciplinas sociales debe partir de un enfoque estructural que explicite sus condicionamientos. Desde la emergencia de las teorías del etiquetamiento quedó claro que la reacción social y por ende los mecanismos de control que la sociedad pone en práctica, son parte de esos condicionamientos.

En las consideraciones de E. Durkheim (1972) la desviación se genera a partir de hacer las reglas o normas por lo que, desde su punto de vista, tal desviación no es "una cualidad" del hecho, la conducta, el comportamiento en sí mismo sino resultante de la perspectiva que está contenida en la regla que elaboraron otros. Tales enunciados alcanzan concreción en el concepto sociológico de anomia que remite a ciertos estados de vacío, a carencia de normas en una sociedad, que provoca entre otras consecuencias, la conducta desviada de algunos individuos (Durkheim, 2003).

La perspectiva durkheimniana aporta sin dudas un elemento relevante al análisis de la desviación, que rompe con las clásicas visiones positivistas, al afirmar que las causas del fenómeno había que buscarlas en la situación anómica y no en las características personales del individuo.

En esta dirección planteó la necesidad de transitar de las impresiones subjetivas a los aspectos estructurales esenciales que están:

[...] en esas maneras de actuar, robustecidas por la práctica, que se denominan costumbres, leyes, usos. Aquí no nos ocupamos de simples incidentes de la vida personal, sino de prácticas regulares y constantes, residuos de experiencias colectivas, que han sido modeladas por toda una cadena de generaciones (Durkheim, 1975, pp.18-19).

No obstante, su análisis funcionalista redundó en la aceptación de una supuesta positividad de la desviación, y en particular del crimen, sesgado por un enfoque esencialmente conservador. Ha sido esta una de las contradicciones que ha transversalizado el análisis de la desviación y todas las categorías asociadas a esta en el pensamiento social. En particular desde la Sociología la contraposición consenso-conflicto ha permeado las consideraciones sobre la desviación, que se debaten entre una visión 
refuncionalizadora del orden establecido y la enunciación de los males acarreados por el advenimiento de la modernidad.

La conflictividad de la condición desviante latente en la indisciplina social no puede ser entendida en términos simplistas como mero desajuste o ruptura con el orden establecido. Sin dudas son atributos asociados a aquellos comportamientos etiquetados como indisciplinas sociales pero su conflictividad no es más que expresión de lo que constituyen fenómenos comunes a cualquier tipo de sociedad.

\section{El control social, su mediación en las ideas sobre indisciplina y la desviación social}

El comportamiento al interior de la sociedad está regido por normas. Los grupos están constantemente poniendo en juego sus reglas, pautas de comportamiento que regulan el actuar en su interior. El control se hace latente hasta en las más sutiles expresiones, como garante del cumplimiento de esas normas.

Todas las sociedades poseen estructuras de control social, mecanismos que le posibilitan su funcionamiento, su mejor desenvolvimiento y que dan lugar a la permanencia de la vida de la sociedad. Estos elementos sirven para imponer un determinado orden social con su correspondiente institucionalidad, son los encargados de mantener la estabilidad, evitando, o al menos restringiendo, las conductas inapropiadas que pudieran expresarse en la misma.

De una u otra forma en estas definiciones se hace referencia a las dos variantes del control social, el informal y el formal. Expresiones dicotomizadas del control que se han configurado a lo largo del devenir histórico de esta categoría en el pensamiento social. Ambos constituyen tipos ideales del control que perpetúan la lógica metafísica de fragmentación de la realidad.

Se legitima así en los discursos sobre el control la impostergable necesidad del orden como punto de partida y fin en sí mismo. De ahí que el escenario queda preparado para una transición en la que los mecanismos de control no se conciben como procesos mediadores para el análisis de las 
contradicciones que a nivel social se expresan en las llamadas desviaciones, sino como instrumentos por excelencia para ejercer coercitivamente una acción correctora sobre las conductas individuales. En otros términos, se está ante la contradicción que representa el orden social respecto a la inserción y la integración social desde el disciplinamiento. Como apunta Carballeda (s/f) lo importante es cómo se establece el orden y cómo se logra la conformidad con el mismo.

Las comportamientos de los diferentes sujetos y grupos sociales son sancionados o gratificados en dependencia de lo que esté legitimado socialmente como aceptado. La conducta desviada aparece entonces como una expresión de desajuste social, un comportamiento que manifiesta incumplimiento de las normas sociales y legales establecidas, que también viene estrechamente vinculada con el término indisciplina social.

Estos procesos clasificatorios pretenden reducir la sociedad a un conjunto de indicadores por medio de los cuales debe identificarse a cada sujeto, sin tener en cuenta la relación dialéctica entre individuo y sociedad. A partir de este enfoque se define el contenido del control, como el proceso que modula el comportamiento estándar de un sujeto que se corresponda con los intereses de la sociedad.

Se expresa así el tratamiento homogeneizante que a su vez se pretende invisibilizar desde un supuesto reconocimiento de la diversidad, que se traduce en la llamada atención diferenciada y no es más que la perpetuación de la lógica clasificatoria (Fabré, Riera, et. al, 2016).

La distinción entre lo formal y lo informal del control es solo muestra de la tendencia a la politización del discurso científico. Esta postura se consolidó con el desarrollo de la Sociología académica norteamericana y ha gozado de muy buena acogida en el ámbito jurídico penal. Ello debido a que se corresponde con el énfasis en los mecanismos coercitivos del control en supremacía respecto a los de carácter persuasivo. 
De esta manera se esboza una ficticia separación entre lo formal y lo informal del control que redunda en definirlo no por su naturaleza intrínseca de acuerdo a su esencia dinamizadora, sino por sus expresiones estereotipadas que lo constriñen en los estrechos marcos del poder represivo.

Desde uno u otro punto de vista el control ya sea desde una perspectiva formal o informal se sustenta para su realización en la interiorización por parte de los sujetos, de las normas de la sociedad, lo cual históricamente ha estado asociado con la socialización y la educación.

\subsection{Educación cívica, socialización e indisciplina social}

La indisciplina social debe verse ligada a la formación cívica o ciudadana de los miembros de una sociedad concreta. Aquellos comportamientos fuera de lo legitimado socialmente son, en gran medida manifestaciones de una carente formación cívica o ciudadana de manera que existe una relación entre educación cívica como medio de socialización coherente con el ideal social y la indisciplina social (Hernández, 2003).

Si como afirma Touraine (1993),

La vida de una Sociedad, se basa en la interiorización de normas, la correspondencia entre las instituciones que elaboran y hacen respetar las normas y las instituciones encargadas de socializar a los miembros de la colectividad, especialmente a los recién llegados, niños o inmigrantes. (p. 345)

Entonces, de esta manera se pone en marcha, por parte de la sociedad, intervenciones preventivas que evitan desajustes de las conductas y responden a un funcionamiento social ajustado a las normas cívicas transmitidas a partir de la socialización, asumiendo una función preventiva.

En las primeras reflexiones con relación a la formación ciudadana aparece la idea de educar al individuo en sus relaciones con el Estado, haciéndolo cumplir sus compromisos con él y también reconociendo cuáles son los derechos que posee con relación a este.

No obstante se encuentra un pensamiento más acabado que no limita sólo la enseñanza cívica a los temas asociados al Estado. Rompe con el pensamiento precedente que entendía a la educación cívica estrechamente ligada a los deberes y derechos ciudadanos. La concepción del Dr. Alfredo M. 
Aguayo (1943) revoluciona los criterios pedagógicos existentes hasta el momento porque asume una concepción desarrollada, amplia que incluye la intensidad de la socialización y la integralidad de los conocimientos: "La educación para la democracia exige, pues, una intensa socialización del individuo. No es una disciplina bautizada con el nombre enseñanza cívica, sino una educación de índole integral que se dirige a todos los aspectos de la vida del alumno" (Como se cita en Sáez, 2001).

Más adelante se aprecia una expansión de la educación cívica hasta alcanzar dentro de la reflexión una nueva connotación, ampliando sus objetivos y alcances.

En este sentido Sáez (2001) señala:

La convivencia social, como el modo civilizado de relacionarse los miembros de una comunidad cívica determinada a partir de la observancia de las normas jurídicas y morales, ha sido y es uno de los objetivos básicos de la educación cívica en cualquier parte del mundo. (p.40)

Es posible derivar a partir de los diferentes criterios que a lo largo de los años se han establecido sobre qué entender por educación cívica, que la misma ha sufrido durante su existencia, un grupo de transformaciones significativas que han devenido en modificaciones estructurales de la enseñanza cívica, no obstante sus objetivos se mantienen como expresión del mantenimiento y organización social.

De lo anterior se deriva como aspecto limitador que la educación cívica haya sido concebida en Cuba, solo como una materia, una asignatura más en el currículo y no como un proceso integrador, donde además de los maestros participa el resto de la sociedad. Esta idea explica que quienes más se hayan interesado en este sentido sean precisamente pedagogos. El hecho de que la educación cívica haya sido abordada, fundamentalmente desde la óptica pedagógica, limita su alcance, dejando fuera otros agentes contribuyentes al proceso.

Se señala que aunque para la formación de los actores sociales el ámbito escolar ejerce una fuerza reconocida, ya sea a través de los conocimientos ofrecidos en las materias impartidas o producto de la exposición 
a los procesos de socialización la educación cívica no debe limitarse a dicha institución, porque los sujetos en su accionar cotidiano interactúan con la sociedad en su totalidad.

Todos los integrantes de una colectividad están obligados a contribuir, desde su posición, al mantenimiento del orden y la estabilidad social, en que la primera labor es enseñarles a los individuos los modos de proceder, mostrando las conductas apropiadas y corrigiendo aquellas que así lo necesiten.

Tanto la educación cívica como la socialización tienen como objetivo supremo la promoción de relaciones sociales estables y armónicas en la sociedad, permitiendo la cohesión y la integración de todos sus miembros, así, también previenen la generación de desajustes socialmente relevantes.

Es posible entender entonces la intrínseca relación existente entre educación cívica y socialización, lo que se traduce en un aspecto importante de la prevención social, cuyo marco referencial de origen son las normas morales, jurídicas y sociales imperantes en la sociedad de la cual forma parte la comunidad concreta (Sóñora, 2006).

Educar a hombres y mujeres de una sociedad como ciudadanos, va mucho más allá de la mera instrucción académica. Implica fomentar en estos sujetos el respeto por el otro, el sentido de nacionalidad, la identidad con aquello que los distingue, con sus raíces, su historia y su cultura. Sobre todo implica conocimiento de todo aquello que como ciudadanos los une, de sus deberes y derechos y de esa responsabilidad moral que se concreta en cada uno de los roles que asumen como parte de la dinámica socio estructural que comprende a toda sociedad.

Un individuo que conoce las contradicciones de la estructura social a la que pertenece, y por tanto está en condiciones de enfrentar su solución, es un individuo con conocimiento de causa y libre. Esta libertad posibilita el desarrollo del protagonismo personal y social en la solución de los problemas que a él le plantea la vida cotidiana (que son al mismo tiempo resultado, o manifestación de dichas contradicciones). Esto a su vez contribuye a que desarrolle una personalidad autónoma, consciente y coherente en el ejercicio de los diferentes roles sociales, asignados-asumidos a través de la práctica histórico social. 


\section{Indisciplinas sociales. Algo más que una excusa}

El control social, la normatividad y el orden asimilado (desde la comprensión de Bourdieu habitus ) son condicionantes que en tanto tales limitan y restringen la mirada culta hacia la cuestión social y, por consiguiente, se obstaculiza la comprensión del orden y la integración social así como la producción de nuevas maneras de afrontar la problemática de la indisciplina social. Es por tanto inexcusable trascender las apariencias para hacer las lecturas correctas de las esencias, de la génesis social de tal estado de cosas.

Se impone el reto por tanto, de un análisis que desborde los marcos formales. Concebir a las indisciplinas no desde una visión solamente normativa, reguladora, normalizadora ni defensista sino como el medio para anticipar aquellas rupturas en el orden social que tendencialmente se expresarán en comportamientos desviados o antisociales así como para develar a través de ellas sus condicionamientos. En un sentido más práctico del análisis esto se resume en asumir al problema como parte de la solución, desde una postura crítica que no se iguale a las visiones funcionalistas de la desviación, o de las indisciplinas.

No se trata del examen de la insdisciplina en sí misma, lo que redundaría en una abstracción del fenómeno, sino en comprender que la indisciplina está expresando la dinámica histórica de las contradicciones sociales, que se manifiestan por lo general como contestarias al orden establecido.

Aporta a lo planteando la idea Mills (1996) acerca de que “...el problema del orden, en su formulación más útil, puede llamarse ahora el problema de la integración social. Requiere, desde luego, un concepto básico de la estructura social y del cambio histórico" (p. 62).

Cuba no ha estado exenta de presenciar un conjunto de dificultades que entorpecen el funcionamiento social desde la formación y "maduración de la conciencia nacional" (Guadarrama, 1985; citado en Sáez, 2001), aspecto que se considera tiene un peso central para analizar el pensamiento social cubano. A lo largo de nuestra historia insignes intelectuales han estado insertos en este debate, cabe mencionar a Félix Varela, José de la Luz y Caballero, José 
Antonio Saco, Enrique José Varona, José Martí, entre otros. De manera incipiente son reconocidas por estos pensadores aquellas conductas que iban contra el desarrollo y el bienestar de la sociedad cubana.

Cada uno de los textos constitucionales cubanos recoge más o menos explícitamente, de acuerdo a cada contexto histórico, las pautas que definen el deber ser del actuar de los ciudadanos cubanos. Sin dudas estas ideas están mucho más claras en la Constitución de 1940, aquí se presenta como obligación de cada cubano observar la conducta cívica, inculcándola a los propios hijos y a cuantos estén bajo su abrigo. De dicha Constitución se desprenden las ideas esenciales que se han ido perfeccionando a lo largo de los años y que se visualizan en la vigente Constitución de la República de Cuba, la cual fue redactada y proclamada en 1976 y reformada en 1992.

La idea con relación a la indisciplina social a través de los años en nuestro país, puede verse asociada a determinados males sociales que han aquejado a la sociedad cubana desde la propia configuración del sentido de la nacionalidad en Cuba y que han venido marcando nuestra identidad a partir de la herencia sociocultural que nos caracteriza, todo lo cual apunta hacia procesos que atañen a la formación ciudadana desde los valores y principios éticos que deben regir en la sociedad.

En diferentes contextos históricos y además debido a la preeminencia de las visiones positivistas sobre el tema, se ha constatado una notable indeterminación de la indisciplina social como concepto orientador de una práctica consecuente y una insuficiente diferenciación con el delito, categoría cuyo tratamiento debe ser otro.

Todo ello impone la necesidad de examinar críticamente algunas de las perspectivas desde las que en nuestro país se ha venido abordando la problemática de las indisciplinas sociales en los últimos años, y su tratamiento por parte de las instituciones vinculadas al entramado normativo legal.

En el año 2008 es emitida por el Fiscal General de la República de Cuba una nueva resolución que regula el accionar jurídico penal en relación al acometimiento de indisciplinas sociales de diversa índole. En dicha Resolución se argumentaba la necesidad de adoptar medidas de carácter jurídico penal 
dirigidas a enfrentar con la energía y rigor requeridos tales manifestaciones (Resolución 10108).

Lo cierto es que el tratamiento teórico-conceptual de la indisciplina social en Cuba ha sido en extremo escaso. De ahí que las definiciones o más bien nociones manejadas al respecto, no pasan de tener un carácter coyuntural y operativo, que redunda en el enfrentamiento, la minimización de sus efectos y la corrección de las conductas de aquellos que incurren en ella.

La propia ambigüedad conceptual denota la indeterminación respecto a la necesidad de indagar en la génesis de aquello a lo que llamamos indisciplinas sociales, y la búsqueda de los procesos que propician su producción y reproducción en el conjunto de las relaciones sociales.

La indisciplina social o cualquier otro término asociado a ella no es otra cosa que expresión de una desviación social, en tanto se corresponda con un comportamiento que verdaderamente agreda los principios y valores justamente establecidos desde el consenso social. Pero más que ir a la búsqueda del disciplinamiento es necesario remitirse al ordenamiento social que sirve de escenario a estas conductas desajustadas y encontrar allí las pautas para una reorganización de la sociedad que sea coherente con las expectativas, necesidades y aspiraciones de todos los que de ella forman parte.

Los vacíos relativos a la normatividad social favorecen los enfoques reactivo-represores, pues no se parte del análisis de las formas diferenciadas de organización; sino de una visión patológica que redunda en la creación de estereotipos y la estigmatización de determinadas conductas y grupos. No se trata de incrementar los niveles de penalización de la vida social, ni de privilegiar las expresiones coercitivas del control como respuesta al incremento de estas conductas.

Es preciso dar cuenta de los mecanismos articuladores que rigen la dinámica social de nuestros días, e insertar en ese debate una perspectiva que incorpore a los diferentes grupos y sectores de nuestra sociedad.

La vulnerabilidad social es sin dudas una de las dimensiones que con mayor énfasis se incorpora en los abordajes de problemáticas como la 
deviación y en particular de las indisciplinas sociales, aunque no siempre desde un enfoque estructural sino desde su configuración grupal entendida como carencias.

En este particular son acertados los criterios aportados por Zaffaroni (1990), a partir de lo que este autor denomina "culpabilidad por vulnerabilidad" y que en nuestro particular, amén de las críticas que pudieran hacerse, pueden ser reelaboradas en función de la selectividad de la que son objeto determinados grupos en la sociedad por parte de las instancias del control.

La configuración estereotipada y etiológica de la indisciplina social se convierte en antesala de procesos de criminalización primaria y secundaria. Autores como Narváez (2013) se refieren a lo que desde la concepción criminológica se define como co-responsabilidad o co-culpabilidad, en un intento por explicitar la responsabilidad de la sociedad en la determinación y el tratamiento de aquellos comportamientos a los que etiqueta como antisociales.

La superación de los marcos reduccionistas desde los que se ha abordado la problemática de las indisciplinas sociales particularmente en los jóvenes, pasa en primer lugar por asumir el tratamiento de este grupo etario no solo desde la vulnerabilidad y el riesgo como grupo social; sino sobre todo desde la potencialidad que constituye en sí misma la juventud como etapa de la vida.

La construcción de la nueva sociedad es de hecho un experimento y solo un hombre consciente, coherente, inteligente y culto es capaz de llevar a cabo las transformaciones pertinentes a un proceso tan profundo y complejo. Este proceso supone considerar la diferenciación real existente entre los sujetos individuales y sociales presentes.

Fomentar el desarrollo de este tipo de hombre en la actualidad, máxime en las condiciones complejas de nuestro país, precisa de políticas científicamente fundamentadas que promuevan adecuadamente el proceso socializador de la personalidad (Fabré; Riera, et. al, 2016).

Esto solo se materializará desde una praxis consciente y comprometida con la reconstrucción del tejido social, para promover relaciones sociales solidarias, cooperativas, participativas que verdaderamente reconozcan a los 
sujetos individuales y colectivos, no como problemas sino como sujetos para la solución; y los haga interactuar como parte del proyecto social en perfeccionamiento.

Un proyecto social emancipador que condiciona desde sus fines el quiebre de cualquier forma de opresión social por tanto, su perfeccionamiento, implica desarrollar medios potenciadores y generadores de relaciones de simetría. En contraposición a las tendencias reproductoras de asimetrías resultantes de prácticas institucionalizadas, propias de un mundo bajo la impronta de la lucha de clases que pretende restaurar las condiciones de opresión capitalista.

La recuperación de la noción integral es el de la dialéctica de la contradicción entre la concatenación del mundo y la autonomía relativa de las partes. Este es un principio consustancial a la crítica del pensamiento social que se centra en la organización de medios para alcanzar fines incuestionables (racionalidad instrumental) sin un cuestionamiento al sentido de los fines ni al significado de lo que ocurre dentro de "la globalidad de la existencia" (Fuenmayor, R. 1994, como se cita en Espina, 2004).

A causa de la imposibilidad real de alcanzar el control total de cualquier sistema social, se produce la emergencia o marginación permanente de formas distintas de socialización, relacionadas lo mismo a sociedades desaparecidas o a variantes de sustitución de la dominante. Por eso la sucesión y superposición de conflictos por controlar el capital cultural, el económico, el político, etc., en y entre campos y habitus culturales diversos, debe ser analizado en la perspectiva de sus procesos de selección (Fernández, 2014).

Es imperativo para lograr este salto cualitativo que se supere además la visión disciplinar de la ciencia, que fuera cimentada por el liberalismo y hegemonizada por el positivismo. Una visión universalista y segmentadora al mismo tiempo que impuso verdades absolutas e incuestionables. Para ello es necesario volver a los fundamentos teóricos y epistemológicos que dieron origen a las Ciencias Sociales y deconstruir la concepción del mundo que le es funcional al sistema de la dominación del capital. 
Ante esas variantes la “...«concepción del mundo» dominante, puede ignorar o aislar tales alternativas; pero en la medida en que éstas son significativas, la función decisiva es controlarlas, transformarlas o incluso incorporarlas" (Williams, 1994, p. 135). Por lo tanto, lo dominante es codificado en la praxis habitual de los sujetos, pero ello es más que la transferencia estática de la dominación al interior de los distintos campos (Fernández, 2014).

\section{Conclusiones}

El debate sobre la problemática de las indisciplinas sociales en la sociedad cubana actual debe rebasar los estrechos marcos del instrumentalismo y las posturas correctoras del comportamiento desviante.

El redimensionamiento de la concepción de las indisciplinas sociales necesita estar transversalizado por la incorporación de cuestiones como lo generacional, el sentido de lo público y lo privado y las pautas socializadoras que definen la llamada convivencia social. Desde esta lógica contribuir al diseño de estrategias y políticas que se atemperen a las características del entramado social cubano y sus disímiles mecanismos de organización social.

El etiquetamiento solo conducirá a la perpetuación de aquellas conductas que asumimos como expresión de indisciplinas.

Las estrategias concebidas desde las instituciones para el tratamiento de las indisciplinas sociales requieren una visión a largo plazo en función de los principios del proyecto social cubano, que prevenga la naturalización de determinados comportamientos ciudadanos identificados como indisciplinas sociales.

La problemática de la indisciplina social está necesitada de ser reflexionada desde una perspectiva multidisciplinar para contribuir a la eficacia de su enfrentamiento a partir de la lógica del desarrollo social emancipador y de la comprensión de este término y su empleo en la intencionalidad de las acciones, que desde la política social de prevención, estructuran los objetivos de dichas instituciones.

El punto de partida para el ejercicio científico investigativo de esta problemática tiene que ser la determinación conceptual de lo que estamos entendiendo por indisciplina social, sin caer en eufemismos o en voluntarismos. 
Es necesario analizar la sociedad, sus estructuras, su organización, sus mecanismos de producción y reproducción y el sistema de relaciones en el que todos estos procesos se concretan.

\section{Referencias Bibliográficas}

Alfonso, G. (2008). Valores y vida cotidiana. La Habana, Editorial Ciencias Sociales.

Alonso, J. et. al. (2004). Autodesarrollo comunitario. Crítica a las mediaciones sociales recurrentes para la emancipación humana. Santa Clara, Villa Clara. Cuba. Editorial Feijoó.

Carballeda, A. J. M (s/f) Del desorden de los cuerpos al orden de la sociedad. Capítulo VIII "Del Orden Social a la nueva fragmentación de la Sociedad". Revista Margen Disponible en http//www.margen.org/libro/index.html\#sumar

Castells, R. (1997) La Metamorfosis de la Cuestión Social., Buenos Aires. Paidos.

Durkheim, E. (1972) Las reglas del método sociológico. La Habana. Editorial Ciencias Sociales.

Durkheim, E. (1975): Introduction á la sociologie de la famille. Textes, Paris: Éditions de Minuit, vol III. [Original en 1888].

Durkheim, E. (2003) La división social del trabajo. Capítulo II y III, en: Hernández, A. (comp.) Historia y Crítica de las teorías sociológicas I. Selección de lecturas. La Habana. Editorial Félix Varela.

Espina Prieto, M. (2004) Humanismo, totalidad y complejidad. El giro epistemológico en el pensamiento social y la conceptualización del desarrollo. Colectivo de Autores La participación. Diálogo y debate en el contexto cubano. La Habana. Centro de Investigación y Desarrollo de la Cultura Cubana Juan Marinello.

Espina Prieto, M. (2003) Pobreza, desigualdad y desarrollo. El rol del estado en la experiencia cubana y sus retos actuales. Ponencia para el Seminario El rol del Estado y la lucha contra la pobreza en América Latina y el Caribe. CLACSO-CROP-FJN. Recife, marzo de 2003.

Fabré, I.; Riera, C. M. et. al. (2016) Monografía Investigaciones sobre delincuencia, control social y prevención desde la concepción de lo comunitario: ejes conceptuales y metodológicos (2000-2015). Santa Clara. Villa Clara. Editorial Feijóo.

Fernández, R. (2014). Simetría social en la producción y consumo de música bailable cubana actual desde la perspectiva del autodesarrollo comunitario. 
Tesis presentada en opción al grado científico de Doctor en Ciencias Sociológicas. Santa Clara. Villa Clara. Cuba.

Fiscalía General de la República. Resolución 10 de 2008.Gazeta Oficial de la República de Cuba

Hernández, R. (2003). ¿Cómo se forma un ciudadano? en Revista Temas. No 35, diciembre. 61-82. Cuba

Limia David, M. (1997). Sociedad Civil y Participación en Cuba. Informe de investigación. Instituto de Filosofía del CITMA. Cuba

Limia David, M. (2002) ¿Vida con Sentido o Sentido de la Vida? Una propuesta desde la ideología de la Revolución Cubana. Consejo de Ciencias Sociales CITMA. [En Soporte Digital] Biblioteca CEC-UCLV

Narváez, R. (2013). Culpabilidad por vulnerabilidad y agnosticismo penal o los muertos que vos matáis. Publicado en Revista de Derecho Penal y Criminología, La Ley, Año 3, No 3, 2013. En soporte digital. Biblioteca CECUCLV.

Sáez, A. (2001) Historia de la educación cívica en Cuba. Tesis en opción al grado científico de Doctor en Ciencias Pedagógicas. Recuperado de http//: Tesis.repo.sld.cu/617/1/tesis doctoral. Universidad de Camagüey.

Ritzer, G. (2006) Teoría sociológica contemporánea. La Habana. Editorial Félix Varela.

Sóñora, M. (coord.) (2006). La prevención en Cuba. En Selección de Lecturas Sobre Sociología y Trabajo Social. Villa Clara. Cuba.

Touraine, A. (1993) Crítica a la Modernidad. Buenos Aires. Editorial Planeta.

Williams, R. (1994) Sociología de la Cultura. Barcelona. Editorial Paidós.

Wright, Mills C. (1996) La imaginación sociológica. La Habana. Edición Revolucionaria.

Zaffaroni, E. R. (1990) La filosofía del sistema penitenciario en el mundo contemporáneo. Conferencia Especial. Seminario Regional para directores de centros penitenciarios y de detención en América Latina, (Cruz Roja Internacional-ILANUD-IIDH, San José de Costa Rica, 14-18 de mayo de 1990). Recuperado el 28 de noviembre de 2015 de: https://inecipcba.files.wordpress.com/2012/10/zaffaroni-la-filosofia-del-sistemapenitenciario.pdf 


\section{Para citar este artículo:}

Riera, C. y Fabré, I. (2017). Las indisciplinas sociales. Una excusa para no mirar allá. Collectivus, Revista de Ciencias Sociales, 4(1), 104-125.

Doi: http://dx.doi.org/10.15648/Coll.1.2017.6 